\title{
Outpatient Myelography: A Prospective Trial Comparing Complications after Myelography between Outpatients and Inpatients in Japan
}

\author{
Tomohiro Matsumoto ${ }^{1}$, Shiro Imagama ${ }^{1}$, Hidenori Inoue ${ }^{2}$, \\ Takaaki Aoki ${ }^{2}$, Naoki Ishiguro ${ }^{1}$, Yoshimitsu Osawa ${ }^{2}$ \\ ${ }^{I}$ Department of Orthopaedic Surgery, Nagoya University Graduate School of Medicine, Nagoya, Japan \\ ${ }^{2}$ Department of Orthopaedic Surgery, Japanese Red Cross Nagoya First Hospital, Nagoya, Japan
}

\section{Study Design: Prospective comparative study.}

Purpose: To compare the incidence and severity of adverse reactions associated with myelography performed in outpatients vs. in inpatients and report the safety and usefulness of outpatient myelography in Japanese patients.

Overview of Literature: Myelography is normally performed as an inpatient procedure in most hospitals in Japan. No studies have reported the usefulness and adverse effects of outpatient myelography in Japanese patients.

Methods: We performed 221 myelography procedures. Eighty-five of the 221 patients underwent outpatient myelography using our new protocol. The incidence and severity of adverse reactions were compared with the other 136 patients, who underwent conventional inpatient myelography. We further compared the cost of outpatient and inpatient myelography.

Results: The overall rate of adverse effects was $9.4 \%$ in outpatients, as compared with $7.4 \%$ in inpatients. Overall, $1.2 \%$ of outpatients and $0.74 \%$ inpatients experienced "severe" adverse effects (requiring hospitalization). There were no significant differences between the 2 groups in either the overall rate of adverse effects or the rate of "severe" adverse effects. Moreover, the average outpatient procedure cost was only one-third to one-half that of the inpatient procedure.

Conclusions: This was the first study to address the safety and usefulness of outpatient myelography in Japanese patients. If selected according to proper inclusion criteria for outpatient procedure, no significant differences were observed in the adverse effects between inpatients and outpatients. The outpatient procedure is more economical and has the added benefit of being more convenient and time-efficient for the patient.

Keywords: Myelography; Complications; Outpatient; Inpatient; Health care costs

\section{Introduction}

Although magnetic resonance imaging has rapidly developed and is currently widely used, myelography is still an essential examination, especially for the dynamic evalu- ation of the spine when diagnosing degenerative lumbar scoliosis in elderly people. Radiological examination of the spinal cord with the use of air myelography was first developed in the early 1900s. Since then, the diagnostic capability and safety of myelography has improved with

\footnotetext{
Received Feb 3, 2015; Revised Apr 29, 2015; Accepted Apr 29, 2015

Corresponding author: Shiro Imagama

Department of Orthopaedic Surgery, Nagoya University Graduate School of Medicine,

65 Tsurumai-cho, Showa-ku, Nagoya city, Aichi 466-8550, Japan

Tel: +81-52-741-2111, Fax: +81-52-744-2260, E-mail: imagama@med.nagoya-u.ac.jp
} 
the development of contrast agents. In the early 1940s, iophendylate was used as a contrast agent, but was not ideal as it was oil-based and caused meningeal reactions resulting in headaches, fever, seizures, dizziness, change of mental status, and adhesive arachnoiditis [1-3]. In the following decades, ionic, water-soluble agents including iocarmate meglumine (CONRAY) and methylglucamine iothalamate became available. However, they were found to be excessively neurotoxic due to their relative hyperosmolarity [3]. In 1972, metrizamide was introduced as a first generation nonionic, water-soluble contrast agent [4]. It was a satisfactory agent, but had a high rate of adverse effects, causing nausea or vomiting in $10 \%-20 \%$ of cases, seizures in $0.2 \%-0.6 \%$, and headaches in $30 \%-50 \%$ patients [2,5]. Because of these persistent side effects, 2 new nonionic contrast agents i.e., iohexol (OMNIPAQUE) and iopamidol (IOPAMILONE) have been developed. These agents cause fewer neuropsychiatric complications than metrizamide, though they have a similar incidence of headache and nausea $[1,6,7]$.

In the early days of myelography, hospitalization was considered necessary due to the relatively high rate of adverse effects after myelography, such as headache, fever, altered mental status, cranial nerve palsy and seizure [1-3]. However, the incidence and the severity of adverse effects has reduced considerably since the introduction of watersoluble contrast media [8], and currently, the procedure is performed as an outpatient procedure in many $\mathrm{X}$-ray departments in most countries [8-10]. However, in Japan, surgeons place enormous importance on the safety of patients, and myelography is routinely performed as an inpatient procedure at most hospitals, even in recent years. There are no reports of experiences with outpatient myelography in Japanese patients. Thus, the aim of this prospective study was to compare the incidence and severity of the adverse reactions associated with myelography in outpatients vs. in inpatients, and to evaluate the safety and usefulness of outpatient myelography in Japanese patients.

\section{Materials and Methods}

All myelography procedures at our hospital were performed on an inpatient basis until 2007. This prospective trial was designed to compare the adverse effects of the inpatient procedure with those of the outpatient procedure that commenced in 2008. The study protocol was approved by the hospitals' Committee on Ethics in Human
Research and informed consent was obtained from each patient.

Between 2008 and 2009, 221 hospital patients required myelography to diagnosis a spinal disorder. Patients who met the inclusion criteria were enrolled for the outpatient myelography procedure, while those who did not underwent conventional inpatient myelography. Inclusion criteria for the outpatient procedure included the following: no severe past medical history; age of $<80$ years; presence of an adult family member on the day of the procedure; and the provision of informed consent acknowledging the potential risks of the outpatient procedure.

Of the 221 patients, 85 patients who met above mentioned inclusion criteria underwent the outpatient procedure, while the remaining 136 patients underwent the conventional inpatient procedure. The outpatient group consisted of 55 men and 30 women, with a mean age of $60.6 \pm 14.1$ years. The inpatient group consisted of 87 men and 49 women with a mean age of $63.5 \pm 14.2$ years. There were no differences in the male/female ratio (not significant [NS], chi-square test) or age (NS, Student's $t$-test) between the 2 groups. The patients' primary diseases were shown in Table 1. Lumbar spinal stenosis was the most common disease, followed by lumbar disc herniation, and cervical spondylotic myelopathy. Patients with cervical spondylotic myelopathy and cervical ossification of the posterior longitudinal ligament tended to present as inpatients rather than outpatients; other than this, there were no significant differences between the groups with regard to primary disease.

All inpatient and outpatient myelography procedures were performed by 1 of 4 orthopedic spine surgeons (each with $>5$ years of experience) using the following procedure: The skin was sterilized in a standard manner, and local anesthesia was administered with several milliliters of $1 \%$ lidocaine. The dural sac was punctured using a 21-gauge Quincke (conventional and normal) spinal needle, usually from the L2/3 to L4/5 level (determined fluoroscopically). Lumbar and cervical myelography was performed by injecting $8-10 \mathrm{~mL}$ of iohexol $240 \mathrm{mgI} / \mathrm{mL}$ (OMNIPAQUE 240, Daiichi-Sankyo Co, Tokyo, Japan).

The outpatient myelography protocol was as follows: Following the myelography procedure, patients were moved to a vacant bed in the orthopedic hospital ward and instructed to rest their upper body at a $30^{\circ}$ angle. The patients were observed for 3 hours while receiving $500 \mathrm{~mL}$ of lactate Ringer's solution intravenously. The 
Table 1. Primary diseases

\begin{tabular}{|c|c|c|}
\hline Disease & Outpatient group ( $\mathrm{n}=85)$ & Inpatient group (n=136) \\
\hline \multicolumn{3}{|l|}{ Cervical spine } \\
\hline Cervical spondylotic myelopathy & 5 & 28 \\
\hline Cervical ossification of posterior longitudinal ligament & 1 & 9 \\
\hline Cervical disc herniation & 4 & 2 \\
\hline Others & 1 & 2 \\
\hline \multicolumn{3}{|l|}{ Thoracic spine } \\
\hline Thoracic ossification of ligament & 1 & 3 \\
\hline Thoracic vertebral collapse from osteoporotic fracture & 0 & 3 \\
\hline Others & 1 & 2 \\
\hline \multicolumn{3}{|l|}{ Lumbar spine } \\
\hline Lumbar spinal stenosis & 40 & 45 \\
\hline Lumbar disc herniation & 21 & 25 \\
\hline Lumbar far-out syndrome & 3 & 5 \\
\hline Degenerative lumbar scoliosis & 2 & 3 \\
\hline Lumbar spondylolysis & 3 & 1 \\
\hline Lumbar spinal tumor & 3 & 0 \\
\hline Lumbar vertebral collapse from osteoporotic fracture & 0 & 3 \\
\hline
\end{tabular}

patients' vital signs and symptoms were recorded immediately following myelography, and at 30 minutes, 1 hour, and 3 hours after the procedure. Three hours after the procedure, the patients were discharged home if no adverse effects were observed. They were instructed to take complete rest, and to refrain from using the toilet at home until the next morning.

The clinical course for the outpatient procedure was documented on a form, with the observation points clearly marked by check boxes (headache, nausea, vomiting, paralysis, seizure, low back pain, or fever) with space to record vital signs and any medications administered. This form ensured rapid, easy and thorough evaluation without the risk of omission.

The inpatient myelography protocol was as follows: After the procedure, patients were moved to a bed in the orthopedic hospital ward and instructed to rest the upper part of the body at a $30^{\circ}$ angle while receiving 500 $\mathrm{mL}$ of lactate Ringer's solution intravenously. The patients remained in the ward until 3 hours after the myelography and were instructed to continue to rest and refrain from using the toilet until the next morning. Vital signs and symptoms were recorded using the same timing as the outpatient procedure up until 3 hours after myelography; after that, patients were monitored every 6 hours until the next morning, at which point the patients were discharged home if no adverse effects were observed.

Both groups were asked to follow-up at the outpatient clinic approximately 1 week after myelography. At this time, the myelographer asked the patient specific questions regarding headache, nausea, vomiting, or other adverse effects. The severity of the adverse effect was subjectively graded by the patient. The severity of symptoms were rated as follows: (1) "not severe," when the patient had some difficulty in performing activities of daily living (ADL), but recovered within several days without hospitalization and (2) "severe," when the patient had marked difficulty in performing ADLs and required hospitalization.

Additionally, we surveyed the cost of outpatient and inpatient myelography in the Japanese health insurance system for procedural cost comparison and its effect on medical economy.

\section{Statistical analysis}

The SPSS ver.20 (IBM, Chicago, IL, USA) was used for statistical analysis. All values were expressed as 
mean \pm standard deviation. An analysis of variance with a Student's $t$-test, chi-square test, or Fisher's exact test was used for comparisons between the groups. A $p<0.05$ was considered statistically significant.

\section{Results}

\section{The outpatient group $(n=85)$}

No adverse reactions were recorded in 78 patients (90.6\%), while the remaining 8 patients (9.4\%) experienced headache $(\mathrm{n}=7)$, nausea $(\mathrm{n}=3)$, and neck pain $(\mathrm{n}=1)$ (Figs. 1, 2). No neurotoxic reactions, such as generalized seizures or neurobehavioral abnormalities, were observed. The symptoms were rated as "not severe" by 7 patients $(8.2 \%)$ who recovered completely within several days after the intake of fluid and rest at home or a steroid drip, if desired by the patient, at the outpatient clinic [11,12]. One patient (1.2\%) experienced "severe" symptoms including a protracted headache and severe nausea that required hospitalization; this patient recovered with rest and intravenous antiemetic drugs, steroid and lactate Ringer's solution (Fig. 3).

\section{The inpatient group $(\mathrm{n}=136)$}

No adverse reactions were recorded in 126 patients (92.6\%), while the remaining 10 patients (7.4\%) experi- enced headache $(n=8)$, nausea $(n=3)$, neck pain $(n=1)$, and dizziness $(\mathrm{n}=1)$ (Figs. 1, 2). No neurotoxic reactions were observed. The symptoms were "not severe" in $9 \mathrm{pa}$ tients $(6.6 \%)$, who recovered completely within several days with the intake of fluid and rest at home or a steroid drip, if desired by the patient, at the outpatient clinic. One patient rated symptoms as "severe" $(0.74 \%)$, as the patient experienced a protracted headache and severe nausea, which required extended hospitalization; this patient

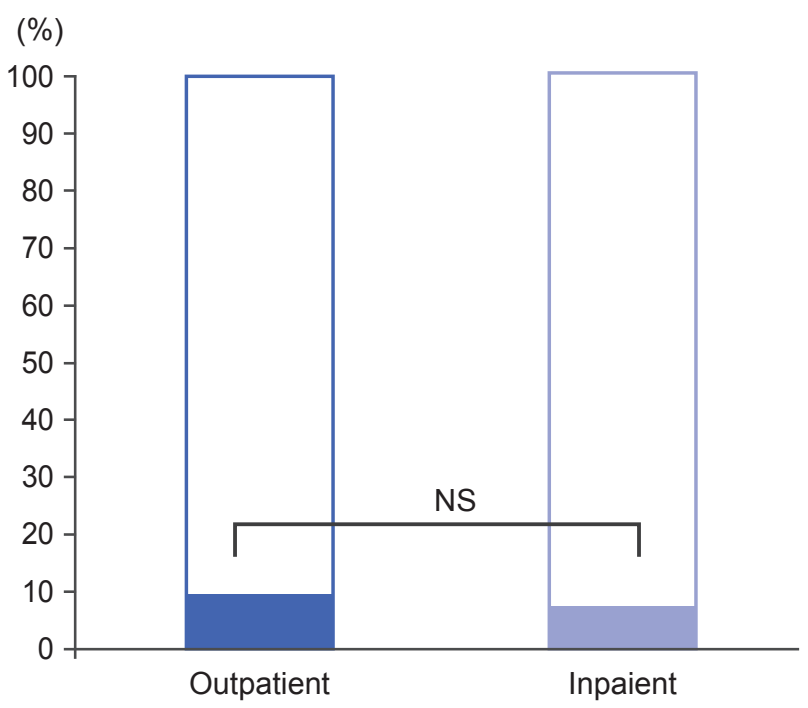

Fig. 1. The overall rate of adverse effects observed in 85 outpatients and 136 inpatients. NS, not significant.

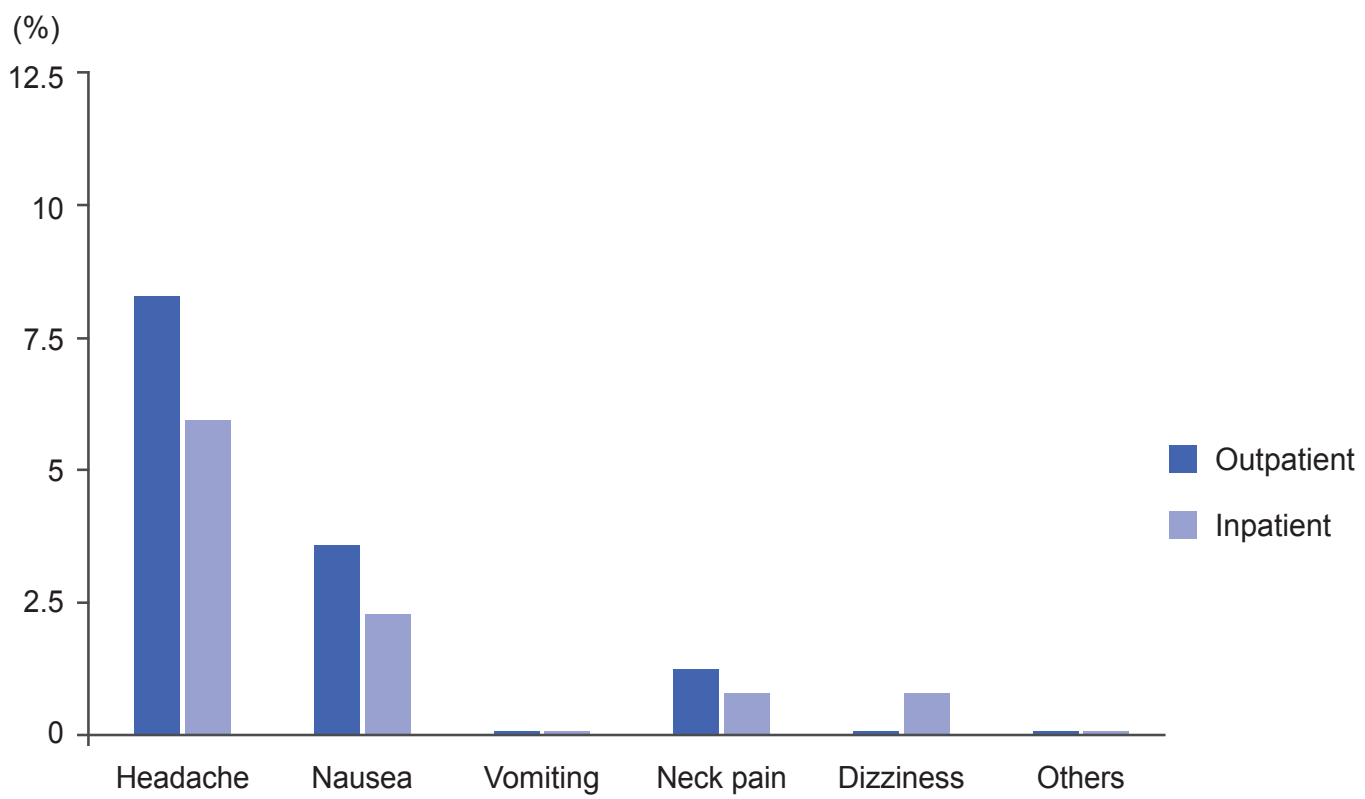

Fig. 2. Types of adverse effects observed in 85 outpatients and 136 inpatients. 
(\%)

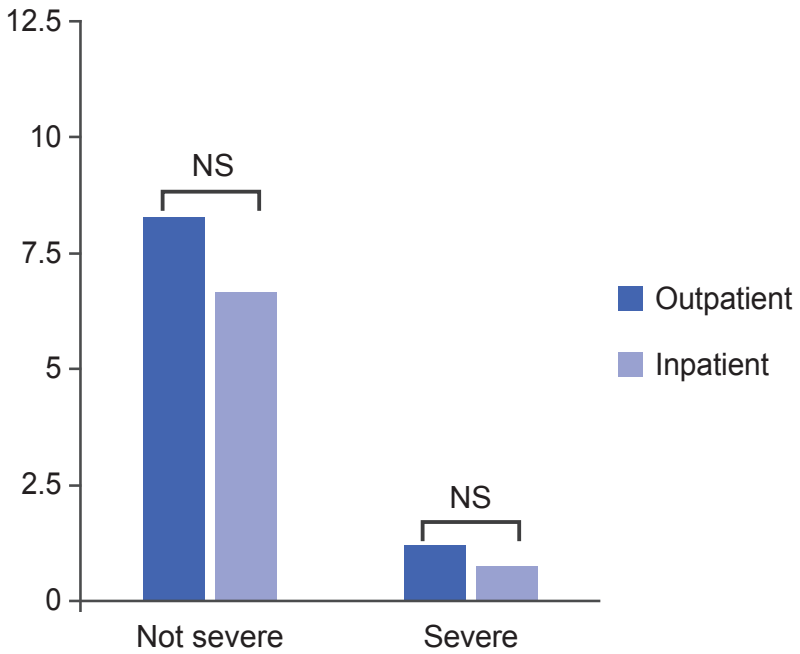

Fig. 3. Degree of adverse effects in 85 outpatients and 136 inpatients. NS, not significant.

recovered with rest and intravenous antiemetic drugs, steroid and lactate Ringer's solution (Fig. 3). There were no significant differences in the rate of steroid injection between inpatients and outpatients.

\section{Comparison between the groups}

The overall rate of adverse effects was $9.4 \%$ in the outpatient group, as compared with $7.4 \%$ in the inpatient group (NS, $p=0.59$; chi-square test). The rate of "not severe" adverse effects was $8.2 \%$ in the outpatient group, as compared with $6.6 \%$ in the inpatient group (NS, $p=0.65$; chisquare test). Finally, the rate of "severe" adverse side effects was $1.2 \%$ in the outpatient group, as compared with $0.74 \%$ in the inpatient group (NS, $p=1.00$; Fisher's exact test). No patients experienced permanent adverse effects in either group.

\section{The cost of myelography in Japanese hospitals}

In the health insurance system in Japan, medical cost is determined according to procedure in a single uniform manner according to the national standard.

Inpatient procedure (hospital stay of 1 night and 2 days): For lumbar herniation (Diagnosis Procedure Combination system), the cost is 7,356 points in the Japanese health insurance system (623 USD). For lumbar spinal stenosis (fee-for-service system), the cost is 10522 points
(892 USD).

Outpatient procedure: For all spinal diseases, 3,126 points in the Japanese health insurance system (fee-forservice system, including all technical fees and drug costs) (265 USD).

\section{Discussion}

In this prospective comparative study, there were no significant differences in the rate and severity of adverse effects between inpatients and outpatients. To our knowledge, this was the first study to discuss the safety and usability of outpatient myelography in Japanese patients.

The safety, cost, and other social factors should be discussed when introducing our new system for outpatient myelography. First, the adverse effects of myelography result from the use of the contrast agent and the lumbar puncture while administering the agent. The adverse effects caused by the contrast agent historically include allergic reactions, nausea and vomiting, anaphylaxis, and seizures. However, the incidence and the severity of adverse effects have reduced considerably since the introduction of water-soluble contrast media (such as iohexol). Post-approval research on OMNIPAQUE (1,8657 cases) indicated that all adverse effects from contrast agents occur at a rate of only $2.2 \%$, which is considered relatively low, as compared to the frequency in past decades [1-3]. Therefore, the contrast agent itself is considered relatively safe in recent years, and it is more important to discuss the complications associated with the lumbar puncture.

The adverse effects resulting from the lumbar puncture are due to leakage of cerebrospinal fluid. These adverse effects include headache, nausea, and vomiting. Preventive countermeasures have already been reported in various studies and include the following [13]: the use of as thin a needle as possible [14-16]; insertion of the needle with bevel parallel to the direction of fiber of the dura; (i.e., parallel to the long axis of the spine) $[15,17,18]$, replacement of the stylet during withdrawal of the needle [19]; and the use of non-cutting (pencil-point type) needle [16]. Definite evidence is available in support of these techniques. However, there is no evidence of the efficacy of increasing fluid intake following the procedure [20]. Previously identified risk factors for headache included young age and female sex $[14,21,22]$. In this study, there was no difference in the male-to-female ratio and age between the groups. Procedures for both groups of pa- 
tients were performed during the same study period, by the same myelographers, using the same techniques and devices. The duration of post-procedure rest was the only difference between the 2 groups; however, there were no significant differences in the complication rates. Our findings were consistent with those of previous studies that found no evidence supporting the efficacy of an increased duration of rest following myelography [23-26]. Thus, the outpatient procedure can be considered safe.

Second, the effects of outpatient procedures on the medical economy should be discussed. As stated above, the outpatient procedure costs only one-third to onehalf as much as the inpatient procedure. Therefore, the outpatient procedure is very economical for patients and may be effective in reducing the cost of medical care in Japan. Additionally, this procedure has the added benefit of being more time-efficient for the patient. Although the inpatient procedure takes a full day (including 1 night stay at the hospital), the outpatient procedure takes only approximately 4 hours.

\section{Conclusions}

This was the first study to discuss the safety and usability of outpatient myelography in Japanese patients. The results of the prospective study suggested that if the patients were selected according to proper inclusion criteria for outpatient myelography, there were no significant differences in the incidence and severity of adverse effects between inpatients and outpatients following myelography. The cost of the outpatient procedure ranged from onethird to one-half of the inpatient procedure and was thus more economical for patients and effective in reducing the cost of medical care in Japan. Furthermore, the outpatient procedure had the added benefit of increasing the number of vacant beds in hospital wards. Additionally, this procedure has the added benefit of being more convenient and time efficient for the patient.

\section{Conflict of Interest}

No potential conflict of interest relevant to this article was reported.

\section{References}

1. Drayer BP, Vassallo C, Sudilovsky A, et al. A double- blind clinical trial of iopamidol versus metrizamide for lumbosacral myelography. J Neurosurg 1983; 58:531-7.

2. Junck L, Marshall WH. Neurotoxicity of radiological contrast agents. Ann Neurol 1983;13:469-84.

3. Olsen J. Seizures after myelography with lopamidol. Am J Emerg Med 1994;12:329-30.

4. Meador K, Hamilton WJ, El Gammal TA, Demetropoulos KC, Nichols FT 3rd. Irreversible neurologic complications of metrizamide myelography. Neurology 1984;34:817-21.

5. Centeno RS, Sovak M, Hackney DB, Garfin SR. Brain changes on computed tomography following metrizamide myelography. Significance and therapeutic implications. Spine (Phila Pa 1976) 1986;11:509-12.

6. Bassi P, Cecchini A, Dettori P, Signorini E. Myelography with iopamidol, a nonionic water-soluble contrast medium: incidence of complications. Neuroradiology 1982;24:85-90.

7. Sobel DF, Rowe R, Zyroff J, Koziol JA, Frost F, Krupsaw J. Adverse reactions to iopamidol and iohexol myelography with special attention to headache: role of myelographic technique. Headache 1989;29:51922.

8. Thorstensen O, Thornorsteinsdottir G, Benediktsson H. Side effects after lumbal myelography: a prospective study of outpatients. Laeknabladid 2001;87:293-6.

9. Aasly J, Anke IM. Experiences with ambulatory radiculography. Tidsskr Nor Laegeforen 1989;109:9512.

10. Pensbo T, Westergaard L. Myelography, can it be performed as an outpatient procedure? Ugeskr Laeger 2002;164:2757-60.

11. Hong M, Shah GV, Adams KM, Turner RS, Foster NL. Spontaneous intracranial hypotension causing reversible frontotemporal dementia. Neurology 2002; 58:1285-7.

12. Pascual LF, Santos S, Escalza I, Iniguez C, MoralesAsin F. Spontaneous intracranial hypotension: quick clinical and magnetic resonance imaging response to corticosteroids: A case report. Headache 2002;42:35961.

13. Evans RW, Armon C, Frohman EM, Goodin DS. Assessment: prevention of post-lumbar puncture headaches: report of the therapeutics and technology assessment subcommittee of the american academy of neurology. Neurology 2000;55:909-14. 
14. Tourtellotte WW, Henderson WG, Tucker RP, Gilland O, Walker JE, Kokman E. A randomized, double-blind clinical trial comparing the 22 versus 26 gauge needle in the production of the post-lumbar puncture syndrome in normal individuals. Headache 1972;12:73-8.

15. Lybecker H, Moller JT, May O, Nielsen HK. Incidence and prediction of postdural puncture headache: a prospective study of 1021 spinal anesthesias. Anesth Analg 1990;70:389-94.

16. Halpern S, Preston R. Postdural puncture headache and spinal needle design: metaanalyses. Anesthesiology 1994;81:1376-83.

17. Norris MC, Leighton BL, DeSimone CA. Needle bevel direction and headache after inadvertent dural puncture. Anesthesiology 1989;70:729-31.

18. Flaatten H, Krakenes J, Vedeler C. Post-dural puncture related complications after diagnostic lumbar puncture, myelography and spinal anaesthesia. Acta Neurol Scand 1998;98:445-51.

19. Strupp M, Brandt T, Muller A. Incidence of postlumbar puncture syndrome reduced by reinserting the stylet: a randomized prospective study of $600 \mathrm{pa}-$ tients. J Neurol 1998;245:589-92.

20. Dieterich $M$, Brandt $T$. Incidence of post-lumbar puncture headache is independent of daily fluid intake. Eur Arch Psychiatry Neurol Sci 1988;237:194-6.

21. Kuntz KM, Kokmen E, Stevens JC, Miller P, Offord KP, Ho MM. Post-lumbar puncture headaches: experience in 501 consecutive procedures. Neurology 1992;42:1884-7.

22. Solomon GD, Clark JW, deSenanayake P, Kunkel RS. Hypersensitivity to substance $\mathrm{P}$ in the etiology of postlumbar puncture headache. Headache 1995; 35:25-8.

23. Dieterich $M$, Brandt $T$. Is obligatory bed rest after lumbar puncture obsolete? Eur Arch Psychiatry Neurol Sci 1985;235:71-5.

24. Cook PT, Davies MJ, Beavis RE. Bed rest and postlumbar puncture headache: the effectiveness of 24 hours' recumbency in reducing the incidence of postlumbar puncture headache. Anaesthesia 1989;44:38991.

25. Spriggs DA, Burn DJ, French J, Cartlidge NE, Bates D. Is bed rest useful after diagnostic lumbar puncture? Postgrad Med J 1992;68:581-3.

26. Hafer J, Rupp D, Wollbruck M, Engel J, Hempelmann G. The effect of needle type and immobilization on postspinal headache. Anaesthesist 1997;46:860-6. 